\title{
HELP YOUR IMPLANT PATIENTS IMPROVE THEIR ORAL HEALTH
}

To combat plaque and other oral health issues Waterpik International, Inc. produce a range of innovative and highly effective Water Flossers including the Waterpik Nano, Cordless Plus, and Ultra models - all of which are supported by a weight of scientific evidence.

Waterpik Water Flossers are proven to be up to $50 \%$ more effective than string floss for reducing gingivitis and are also are up to $29 \%$ more effective at removing plaque from interproximal tooth surfaces.

There are now more Waterpik Water Flosser options than ever for clinicians to recommend to implant patients.

Visit www.waterpik.co.uk.

\section{LEARN IMPLANT RESTORATION AT YOUR OWN PACE}

With the Esthetic Alliance Program (EAP) from Nobel Biocare, you can introduce a new business stream into your practice. You do not need to have prior implant experience as you will receive full training and support in dental implant restoration.

Small groups inspire learning and help to quickly build confidence. Each EAP session has no more than ten dentists, who are taught by a highly experienced surgeon. Participants are required to bring their own cases right from the very beginning. They can learn at their own pace and progress only when they are ready to move on to the next stage.

Programme facilitator Nobel Biocare also assists GDPs to develop referral networks and market the new service to patients. Together with attractive equipment packages on offer, you can begin to help patients without delay.

Earn verifiable CPD while increasing your knowledge, skills and business potential with EAP from Nobel Biocare.

For information on participating in the EAP as a referring GDP or surgeon host, contact Nobel Biocare on 0208756 3300 or visit www.nobelbiocare.com.

\section{AN IMPLANT SYSTEM THAT DOES IT ALL}

The design of ASTRA TECH Implant System EV is based on natural dentition, using a site-specific, crown-down approach to help ensure a successful outcome.

The versatile OsseoSpeed EV implant designs have a wide range of shape, length and width options. This allows the dentist to easily manage and adapt to different challenges as they arise.

The drilling procedure is made easy by using colour-coding and a simple numbering system. The options within the drilling protocol help ensure correct preparation of the marginal bone and allow the preferred level of primary stability to be achieved.

ASTRA TECH Implant system EV has only one surgical tray with three interchangeable overlays, so the content can be set up according to clinical preference. The colour-coded tray has an intuitive layout, for ease of use and effective handling throughout the surgical procedure. The system offers an extensive restorative assortment, including

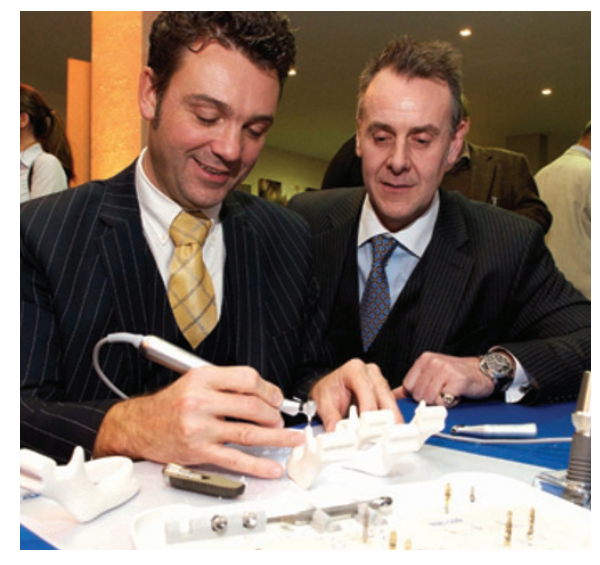

patient-specific abutments and a wide range of pre-fabricated abutments. The components are designed to help support all clinical situations and soft tissue sculpturing requirements for final restorations. They are available in a choice of materials to support the planned final restoration and aesthetic demands.

For further information on ASTRA TECH Implant system EV, email implants. uk@dentsply.com or visit www.dentsplyimplants.co.uk. 